Teknokultura. Revista de Cultura Digital y Movimientos Sociales

ISSNe: $1549-2230$

http://dx.doi.org/10.5209/TEKN.62307

\title{
Identidades colectivas, prácticas artísticas y biodiversidad: los casos de Cerezales y Valldaura ${ }^{1}$
}

\author{
Lorena Lozano ${ }^{2}$
}

Recibido: 7 de noviembre de 2018 / Aceptado: 16 de marzo de 2019 Open peer reviews

Resumen. La deslocalización de la economía y la ubicuidad de la producción son algunas de las consecuencias de la globalización y de las nuevas formas de comunicación, fenómenos que no son ajenos a la producción artística. De hecho, la proliferación de diversos proyectos culturales en las periferias rurales de la Península Ibérica constata que la ciudad y lo urbano ya no son los únicos ámbitos donde se desarrollan las manifestaciones artísticas más relevantes. Muchas de estas iniciativas nacen de la simbiosis entre colectivos artísticos, movimientos sociales y/o instituciones. Su creciente interés por la biodiversidad cultural y su manejo de los nuevos medios tecnológicos abre la posibilidad a experiencias locales que pueden ser territorializadas. ¿Qué percepciones de la biodiversidad y de los nuevos medios coexisten? ¿Cómo se vinculan al territorio los diferentes lenguajes implicados y cómo se componen los saberes vinculados a la biodiversidad? ¿Qué relaciones entre género, comunidad y tecnología se establecen? Para responder a estas cuestiones se llevó a cabo un análisis comparativo de la percepción del paisaje de dos comunidades envueltas en iniciativas de creación cultural y artística en las que conviven identidades campesina, artística y activista. Los resultados ponen de relieve la emergencia de identidades colectivas y reafirman la necesidad de vincular lo natural y lo biológico al aprendizaje empírico y a las prácticas culturales. La discusión puja entre lo orgánico y lo digital y propone los lenguajes y prácticas del arte contemporáneo como motores de nuevos sentidos de comunidad y de procesos de construcción colectiva del conocimiento.

Palabras clave: conocimiento científico; conocimiento popular; nuevos medios; territorialización.

\section{[en] Collective identities, artistic practices and biodiversity: the cases of Cerezales and Valldaura}

\begin{abstract}
The delocalisation of the economy and ubiquitous production are consequences of globalisation and of the spread of new forms of communication, and artistic production has not been immune to these phenomena. The proliferation of diverse cultural projects in rural peripheries throughout the Iberian Peninsula confirms that relevant contemporary artistic expression is no longer confined to cities and the urban sphere. Many of these peripheral initiatives are the result of a symbiosis between art collectives, social movements, and/or institutions. An increasing interest in cultural biodiversity and the use of new technologies allows these local experiences to be territorialised. What is the perception of biodiversity and new media in these contexts? How do the different languages involved connect to the territory, and how is knowledge about biodiversity constructed? What relationships emerge between gender, community and technology? In order to explore these questions, a comparative analysis of the perception of the landscape was carried out in two communities engaged in cultural and artistic initiatives that bring together traditional rural, artistic and activist agents. The results highlight the emergence of collective identities and reaffirm the need to connect nature and biological knowledge to
\end{abstract}

\footnotetext{
Econodos, Fundación Cerezales, Valldaura Labs, Parque de Collserola.

Universidad Abierta de Cataluña (España)

E-mai:1lozanoa@uoc.edu
} 
empirical learning and cultural practices. The discussion moves between the organic and digital spheres and suggests that the languages and practices of contemporary art can be the engine for new forms of community and processes for the collective construction of knowledge.

Keywords: new media; popular knowledge; scientific knowledge; territorialisation.

Sumario. 1. Introducción. 2. Metodología. 3. Resultados. El retorno ilustrado. 4. Implicaciones. 5. Conclusiones. Comparando lo incomparable. 6. Agradecimientos. 7. Referencias bibliográficas.

Cómo citar: Lozano, L. (2019). Identidades colectivas, prácticas artísticas y biodiversidad: los casos de Cerezales y Valldaura, en Teknokultura. Revista de Cultura Digital y Movimientos Sociales, 16(1), 109-126.

\section{Introducción}

La deslocalización de la economía y la ubicuidad de la producción son algunos de las consecuencias de la globalización y de las nuevas formas de comunicación, fenómenos que no son ajenos a la creación artística. De hecho, el imaginario industrial occidental donde las relaciones sociales se organizan alrededor del concepto de producción ha dado un giro hacia otro imaginario en el que la "creación" es valorada como un servicio. Desde finales de la década de 1990, el arquetipo de artista sedentario ha ido mutando hacia un nómada profesional que desarrolla proyectos artísticos entretejidos y confrontados con realidades culturales específicas (Rofes, 2003). Además, la reciente proliferación de muy diversos proyectos artísticos dispersos por algunos territorios rurales de la Península Ibérica constata que la ciudad y lo urbano ya no son los únicos ámbitos donde se desarrollan las manifestaciones del arte más relevantes. Muchas de esas iniciativas, itinerantes entre lo urbano y lo rural, nacen de la simbiosis entre colectivos artísticos, movimientos sociales y/o instituciones. Entre algunos de ellos, Nodar Rural Art Lab desarrolla desde 2006 el archivo Binaural/Nodar vinculado a la Red Tramontana, un proyecto europeo de archivística de zonas rurales y de montaña que recoge, digitaliza y publica documentos sonoros y videográficos de los territorios de Viseu y Aveiro, Portugal. Desde 2010, en el estado español, Montenoso, dirigido por Fran Quiroga, se define como una comunidad y un espacio en red que busca modelos de gobernanza, estudio y (re)situación de los montes comunales como paradigma de propiedad en Galicia y Campo Adentro, dirigido por Fernando García Dory, es una iniciativa sobre territorios, cultura e identidad cuyo objetivo es ensayar una estrategia cultural de lo rural a través de la producción artística.

Dejando de lado especificidades, desde la perspectiva de las ciencias sociales, Duxbury y Campbell (2009) entienden este tipo de experiencias como una forma de conciliación de los territorios urbanos y rurales y defienden su impacto social en la comunidad local ya que facilitan la creación de redes globales y puentes entre saber experto y vernáculo. De forma parecida, el intento de estas iniciativas de archivar y redefinir el conocimiento tradicional y la biodiversidad cultural, es, para Douglas (2004), una forma de revaluar las tradiciones más allá de la homogenización que nos llega de los sistemas de control centralizados. Sin embargo, tal y como advierte Hannerz (1998; 1996), para formar un nuevo vínculo social es ne- 
cesario algo más que imaginar la comunidad global. Las iniciativas mencionadas participan en la gestión de la biodiversidad y hacen uso de los nuevos medios de comunicación digital al mismo tiempo que conforman nuevas relaciones de convivencia entre oriundos, visitantes y nuevos habitantes. Desde la óptica del nuevo orden global, podríamos incluso afirmar que, estas prácticas artísticas, ejercen lo que Appadurai llama el "nuevo rol de la imaginación", algo que nace de la yuxtaposición de flujos de gente y de contenidos mediáticos convertidos en formas de trabajo y de negociación fundamental (2001; citado en Moraga, 2012). Pensemos, además, que la conexión de la cultura con el territorio está debilitado y desubicado provocando las más profundas transformaciones de la experiencia del día a día y de la vida a nivel local. Algo que Escobar (1999) achaca a la distorsión del orden simbólico que conecta la gestión directa de la biodiversidad y las relaciones en sociedad, separando las prácticas orgánicas de las sociales, un testigo de la gran promesa de las nuevas lógicas sociales creativas y los regímenes sensoriales de la era digital (Escobar, 1994, p. 216).

Ante estas circunstancias parece necesario replantear las ya muy estudiadas dicotomías entre saber experto y vernáculo (Benito Lucas, 2013; Boixo, 2002; Eiroa, 2013; Esparcia, 2005; Nogué, 1992; Ortega Cantero, 2009; Pérez Soriano, 2013) y problematizar la confrontación entre las identidades campesina, artística y activista. Cabría preguntarse entonces ¿qué dinámicas se están configurando entre colectivos artísticos, movimientos sociales y/o instituciones culturales en esos medios rurales? ¿Hasta qué punto las prácticas artísticas tienen capacidad para provocar experiencias locales que pueden ser territorializadas? ¿Cuál es su rol? ¿Qué percepciones de los nuevos medios y la biodiversidad coexisten? ¿Cómo se vinculan al territorio los diferentes lenguajes implicados y como se construyen los saberes vinculados a la biodiversidad? y ¿qué relaciones entre género, comunidad y tecnología se establecen? Para responder a estas cuestiones se llevó a cabo un análisis comparativo entre dos comunidades periféricas envueltas en iniciativas de creación cultural y artística en las que conviven diferentes identidades (excampesino/ as, artistas y activistas). El objetivo era estudiar su percepción de la biodiversidad, del paisaje y de los nuevos medios y visualizar las nuevas narrativas que emergen de lo local-global. A continuación, se relatan los métodos de investigación, las estrategias de recopilación de datos, los resultados, el análisis, la discusión y algunas implicaciones derivadas, seguidas de unas conclusiones.

\section{Metodología}

Para llevar a cabo la investigación, se usa el estudio de caso con orientación comparativa como estrategia metodológica y se seleccionan dos muestras de acuerdo a su representatividad dentro de la temática de estudio (Corbin y Strauss, 2008, citado en Vergés, 2012). Durante 2013, en colaboración con la Fundación Cerezales Antonino y Cinia $^{3}$ (provincia de León), desarrollé el trabajo de campo para el primer caso de estudio en un contexto tradicional rural de fuerte carácter local

3 FCAYC desde 2008 es "una institución de carácter privado, orientada al desarrollo del territorio y a la transferencia de conocimiento a la sociedad mediante la producción cultural y la etnoeducación". Recuperado de http:// www.fundacioncerezalesantoninoycinia.org/informacion/fundaci\% $\mathrm{C} 3 \% \mathrm{~B} 3 \mathrm{n}$-cerezales-antonino-y-cinia]. 
bajo presión metropolitana. A través del proyecto Herbarium ${ }^{4}$ participaron en él unas veinte personas mayores de 60 años, en su mayoría mujeres oriundas de la comarca, campesinas y jubiladas que conviven con nuevos habitantes y visitantes de la capital. La comarca es su ámbito de movilidad, se comunican mayoritariamente de forma oral y escrita y no conforman una red establecida. Sus intereses comunes son la identificación y conservación de la flora local, el intercambio de saberes populares y la situación de crisis del entorno rural. Durante 2014 y 2015, en colaboración con las organizaciones Okno (Bruselas) y Valldaura Laboratorios de Autosuficiencia ${ }^{6}$ (sierra de Colserola, Barcelona), desarrollé el trabajo de campo del segundo caso de estudio en un contexto "exagrícola" y postindustrial de la periferia metropolitana. Mediante el proyecto Opengreens ${ }^{7}$ participaron un colectivo de unos veinte creadores, makers, activistas y artistas, en su mayoría hombres entre los 30 y los 50 años, de nacionalidades belga, francesa, portuguesa, checa y también algunos vecino/as catalanes de la sierra. Sus intereses comunes son la gestión de la información, las soluciones tecnológicas artesanales enfocadas al medioambiente y el arte tecnológico inspirado por procesos naturales. Se articulan en red, su comunicación es mayoritariamente virtual y su movilidad es transnacional. El carácter global y deslocalizado de este caso, aunque no contiene los elementos necesarios para poder abordar una compresión local del territorio, resulta imprescindible para encuadrar el objeto de estudio (Fig. 1 y Fig. 2).

Figura 1. Salida de campo de Herbarium, 2013.

Fuente: Fundación Cerezales Antonino y Cinia.

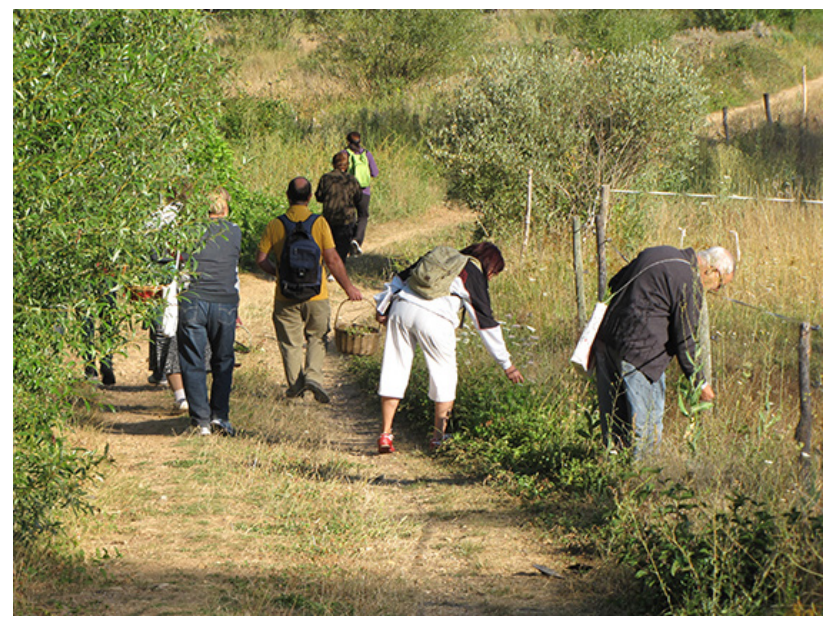

www.herbarium.cc

Okno es una organización de Bruselas que desarrolla proyectos sobre arte y ecología desde el año 2004. [Recuperado de www.okno.be.

6 Valldaura Labs es un centro de investigación del Instituto de Arquitecturas Avanzadas de Cataluña (IaaC) que, desde 2013, une arquitectura, medioambiente y herramientas digitales. Recuperado de http://www.valldaura. net/?lang=es.

7 Opengreens. Recuperado de http://opengreens.net/ 
Figura 2. Grupo de discusión de Opengreens, 2015.

Fuente: archivo de Econodos

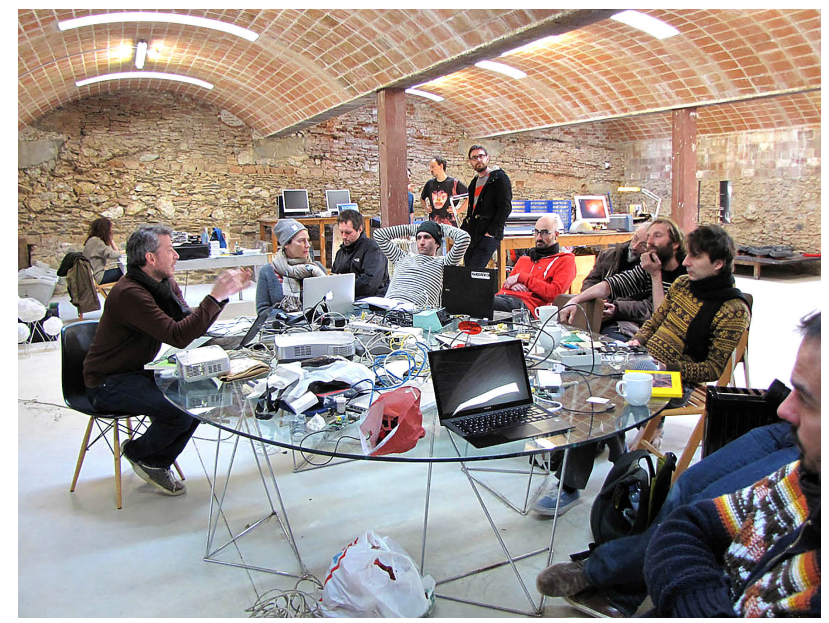

La investigación supuso el desarrollo de un trabajo de campo que consistió en, por una parte, un análisis de ambos contextos territoriales desde el punto de vista de la experiencia espacial humana y de sus dimensiones geográficas, históricas, socioculturales y demográficas (Carrero de Roa, 2010). Para ello se realicé una visualización fotográfica y cartográfica del territorio, la subdivisión espacial del lugar, una matriz de contraste de valores atribuidos al paisaje (ecológico, económico, histórico, espiritual, «identitario» y social) y, finalmente, una matriz DAFO que apunta algunas dinámicas de su evolución territorial. Paralelamente, comencé un proceso de diálogo con los actores sociales basado en técnicas de la etnografía, del análisis del discurso y de la práctica artística (Welpa et al. 2005, Leavy 2008, Yin 2003-2009). Durante esta parte del trabajo de campo organicé encuentros con los participantes de ambos proyectos en los que llevamos a cabo actividades inspiradas y adaptadas de Lévi-Strauss (2002), Luginbühl (2008, pp. 143-180), Lynch (1960, p. 9) y Thapalyal (2005).

Estas dinámicas consistieron principalmente en entrevistas a algunos individuos representativos de la comunidad y talleres grupales, todo ello fue documentado de forma audiovisual y gráfica. Las entrevistas estuvieron enfocadas a los vínculos con la tierra y los cultivos, los cambios en el paisaje, los usos medicinales de las plantas, las relaciones vecinales y de convivencia, su interés por temas ambientales y sus opiniones sobre cuestiones éticas y estéticas derivadas del uso de las nuevas tecnologías. Algunos encuentros consistieron en salidas de campo, recolección de plantas y posterior identificación, caracterización y representación artística mediante dibujo y estampas. Otros talleres, mediante una serie de plantillas prediseñadas y debates posteriores, ayudaron al reconocimiento individual y colectivo de la percepción y los arquetipos del paisaje y el sentido del lugar. Para ello, por una parte, se ofrecieron imágenes icónicas del paisaje a las que se pedía atribuir valores como comodidad, belleza, libertad, salud, fealdad, incomodidad, abandono, etc. El sentido del lugar y la percepción espacial fueron explorados mediante la elaboración de una cartografía "psicogeográfica" del entorno, en la que, sin buscar la habilidad artística, pedí realizar una descripción gráfica 
y subjetiva del mismo (Fig. 3). Con objeto de explorar el significado social de ciertos objetos tecnológicos y de los nuevos medios de comunicación, presenté una serie de artefactos tecnológicos digitales y analógicos procedentes de diferentes contextos culturales (alta/baja tecnología, cultura popular, domesticidad, aperos del campo, tecnología punta, etc.). El ejercicio consistió en describir cada objeto sin referirse a su nombre, función o propósito, tratando de elucidar sobre la cultura de procedencia mediante asociaciones personales o memorias (Fig. 4).

Durante los procesos pude explorar los valores estéticos del paisaje y de la biodiversidad y su relación con los medios tecnológicos ahondando en las subjetividades y asociaciones psicológicas y sentimentales de ambas comunidades. También abordé las formas mediante las cuales manifiestan la relación entre los mundos humanos y biológicos, sus clasificaciones y lenguajes. El análisis de los datos obtenidos a lo largo de todo este proceso me permitió comprender, representar y analizar los esquemas mentales de la comunidad, las relaciones de identidad con el lugar, su percepción de la biodiversidad y su relación con los nuevos medios tecnológicos ${ }^{8}$.

Figura 3. Mapa subjetivo realizado por una de las mujeres de Herbarium, 2013.

Fuente: archivo de Econodos

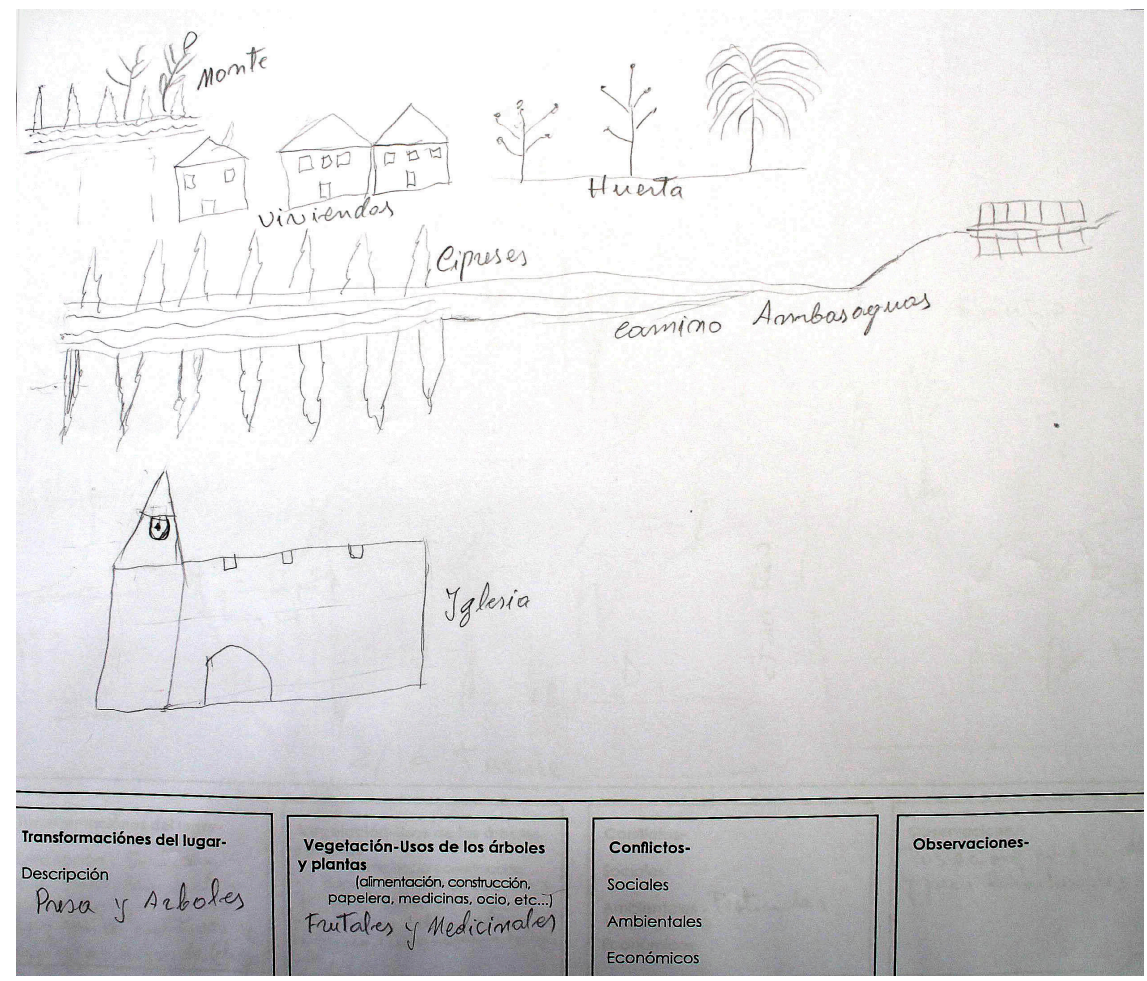

8 Este estudio forma parte de la tesis de doctorado Lozano, L., Jardin en red. Arte, ciencia y sociedad. Universidad de Oviedo, Dpto. Historia del Arte y Dpto. de Sociología. Dir: Natalia Tielve. Co-Dir: Holm Khöler, 2017. Proyecto financiado por Universidad de Oviedo, Asociación Econodos. Ecología y comunicación y Fundación Cerezales Antonino y Cinia. 
Figura 4. Resultados de un cuestionario sobre la percepción de tecnologías digitales y analógicas de un miembro de Opengreens, 2015. Fuente: archivo de Econodos.

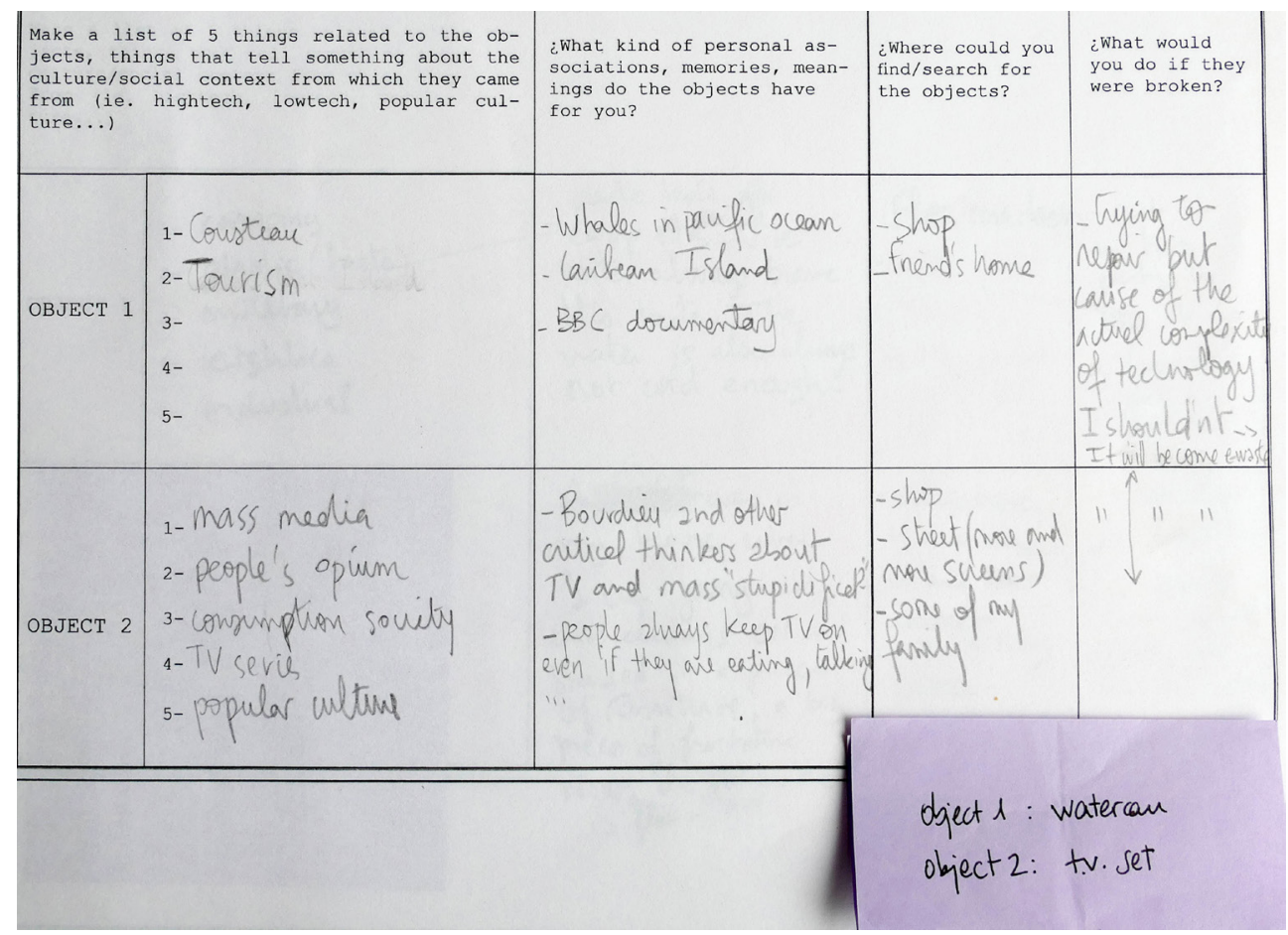

\section{Resultados. El retorno ilustrado}

El estudio nos sitúa, por un lado, en una comarca rural de Castilla y León marcada por el éxodo, el envejecimiento y la masculinización de la población, circunstancias que hacen difícil el mantenimiento de la estructura social. Además, el abandono de las prácticas agrícolas, las políticas agrarias de la UE (PAC) y la desaparición de los Concejos de Vecinos amenazan el poder de gestión del medio por parte de la sociedad civil y debilitan aún más la ecología del medio. El trabajo de campo muestra que, de forma general, el grupo comparte una actitud conservacionista y protectora hacia el patrimonio y el conocimiento popular. Los avances tecnológicos y herramientas aplicadas al campo provocan tensión ya que son percibidos como avances y oportunidades por los oriundos y como contaminantes por los nuevos habitantes. Los sistemas de comunicación digital están siendo lentamente apropiados ya que no satisfacen sus necesidades más inmediatas. Son conscientes de que la visión cosmológica tradicional del mundo rural deja de transmitirse de forma oral o escrita y se convierte en un conocimiento, quizás anacrónico, que posee un soporte difícil de compatibilizar con lo que ofrece el mundo actual y las nuevas formas de relación social (Fernández-Catuxo, 2014). También se pone en evidencia que los significados del territorio de los visitantes y jubilados recién mudados poseen una imagen del lugar alimentada de re- 
latos familiares y memorias, de literatura de guías, atlas e informaciones de mercado o institucionales, entre ellas de la Fundación Cerezales Antonino y Cinia ubicada en la comarca. El campo para ellos es sinónimo de aire puro y tranquilidad y reivindican la protección del paisaje en sus rasgos icónicos, valorándolo en base a modelos del paisaje romántico. En sus mapas marcan hitos geográficos desde donde se pueden observar vistas panorámicas del paisaje. De forma diferente, los oriundos asocian el paisaje a vivencias comunes con otros vecinos y reflejan la relevancia de la actividad agropecuaria como sustento de la vida rural tradicional. Representan el río en sus mapas como eje que configura la ordenación del territorio y son muy conscientes de las grandes transformaciones del paisaje. Señalan aspectos climáticos, hidrológicos, de parcelación, vegetación y cultivos. Perciben el despoblamiento y los cambios del entorno con pesimismo y desarraigo mientras expresan nostalgia ante el abandono, algo que Fredes Álvarez, una de las vecinas expresa así:

- Aquí no sacabas para vivir... a las ciudades, a la industria... Nosotros marchamos ya con treinta y tantos años, mi hijo mayor ya tenía ocho años y la chica cinco años, cuando marchamos de aquí, oye, no veías porvenir, luego salen los chicos arriba, no tienes pa' mandarles a estudiar, porque no tienes ni pa' vivir, o sea que hay que emigrar a donde podamos ganar la vida [risas] y por eso nos fuimos pa' Bilbao.

- Claro, ¡cómo no lo vas a notar! lo han puesto todo patas arriba... yo ya no sé cuáles son los nombres, antes en el campo sabía cómo se llamaba todo, ahora ya no sé nada, ya no sé las divisiones de un lado y de otro, sí, mucho cambio... Al marchar de aquí pierdes todo el contacto.

Estos resultados constatan la dicotomía que mantiene el significado simbólico de la ruralidad, algo característico del contexto español (Eiroa, 2013). Se observa una falta de sintonía entre la identidad de los oriundos y la imagen que proyectan los foráneos, mientras los primeros comprenden el paisaje como un recurso de subsistencia que depende de los conflictos internos y la memoria social, la imagen proyectada por el recién llegado es alimentada por guías de viaje y modelos de paisaje romántico. Esta idealización de lo rural que llega de la ciudad descansa en un canon de belleza paisajística, bucólica y pintoresca de fuertes connotaciones patrióticas y nacionalistas que, en España, bajo la influencia del ideario liberal y reformista de la Institución Libre de Enseñanza (1876 y 1936), la geografía de Humbolt (1769-1859) y los escritores y pintores de la Generación del 98, identifica el paisaje castellano con el paisaje del pueblo español (Nogué 1992; Cantero 2009). Frente a este "idilio rural", los oriundos manifiestan un sentimiento de pesimismo y desarraigo que tiene su origen en la década de 1950 cuando las escuelas rurales, las agencias de desarrollo agrario y la prensa indujeron ideas de menosprecio hacia la tierra, las herramientas y el trabajo tradicionales (Boixo, 1999). Esto supuso la identificación de lo urbano y del éxodo con éxito personal y prestigio cultural provocando la llamada "huida ilustrada" hacia la ciudad, un fenómeno marcadamente femenino (Pérez Soriano 2013; Benito Lucas 2013).

En la otra punta del universo, en la sierra de Collserola nos encontramos en un puerto de montaña ocupado por segundas residencias, espacios de ocio y centros de investigación y empresas que atraen población local e internacional y mantienen una estructura social diversa y dinámica. La figura de Parque Natural y las leyes del pai- 
saje de Cataluña que favorecen las prácticas agrícolas y la "masovería" (aparcería) tratan de proteger la fragilidad ecológica del medio. El trabajo de campo muestra que, a pesar de las diferentes raíces identitarias, el grupo mantiene un vínculo con el mundo rural relacionado con la infancia y los recuerdos familiares. Consideran el parque y los espacios naturales como una parte esencial del patrimonio de la humanidad que proporciona experiencias y servicios recreativos y culturales. Defienden su conservación y protección ya que aprecian la naturaleza como fuente de bienestar y salud. Las vecinos/as de Valldaura entrevistados conciben la sierra como espacio de encuentro social y educativo, de comidas campestres y asociaciones excursionistas, de naturalistas amateur y estudiosos. La fuerte presencia de movimientos sociales, el mantenimiento de la "masovería" y un sentimiento nacionalista catalán que asocia paisaje y patrimonio son rasgos identitarios que revelan su implicación como ciudadanos activos en la gestión del territorio. De forma diferente, el colectivo de makers asocia las tecnologías analógicas al placer del campo y a memorias de la infancia, mientras que las nuevas formas de comunicación las relacionan con la amistad y la libertad. Los paisajes arquetípicos poco urbanizados son relacionados con actividades al aire libre o deportivas y evocan tranquilidad y descanso. Señalan como lugares utópicos y perfectamente sostenibles jardines y granjas autosuficientes, las cuales describen con todo tipo de detalles técnicos. Annemie Maes, artista visual de Bruselas expresa su relación con la biodiversidad de esta manera:

Mi interés por la naturaleza surgió hace mucho tiempo, provocado principalmente por las estructuras matemáticas en la naturaleza: la colmena de las abejas, la red de una araña, la estructura de una hoja de la planta... Lo que yo trato de hacer como artista es «hacer lo invisible, visible». La naturaleza tiene muchas buenas soluciones que ofrecer. Estoy muy interesada en «biomímesis», de cómo podemos aprender de la naturaleza. Como artista, mi principal tema está trabajando con las abejas. Yo las observo y hago visualizaciones artísticas de estas observaciones. Estudio también los campos de búsqueda de alimento, las plantas y las flores melíferas, la naturaleza es una parte intrínseca del medioambiente de las colonias de abejas. El estado preocupante de nuestro medioambiente es una de las principales razones de la desaparición de las colonias de abejas. Es importante crear conciencia sobre este tema para el público en general.

El colectivo de makers construye de forma artesanal representaciones, soluciones tecnológicas y artefactos para la gestión y documentación de cuestiones ambientales. Su filosofía se basa en la apropiación y manejo de los nuevos medios tecnológicos como una oportunidad para la gestión, la investigación y la democratización de los recursos y la búsqueda de soluciones a cuestiones ambientales, la producción de conocimiento y la autorganización. Están motivados por un espíritu conservacionista y al mismo tiempo transformador, una suerte de reinvención del paisaje o «retorno ilustrado» que les hace deudores de la cultura de la Escuela Flamenca del siglo XV, de sus avances técnicos basados en la perspectiva, la topografía y la cartografía. Las características interdisciplinares, "multitécnicas" e interculturales son su respuesta a un momento histórico que pone sobre la mesa nuevas formas y medios de comunicación. Las diferentes identidades creativo, artista, "maker", ambientalista y nacionalista que confluyen en este caso otorgan 
un gran valor a los paisajes naturales y los consideran vehículos de inspiración, de desarrollo artístico y fuente de curiosidad y aprendizaje. Todos reconocen el idilio rural mencionado, pero también afloran rasgos identitarios del "medioambientalismo" en la actitud de defensa del espacio, la comunidad, la autonomía y la reivindicación de una política verde y de una mejor calidad de vida. Su aspiración a corregir las relaciones destructivas entre acción humana y entorno natural son valores universales en los que subyace la identidad biológica o «identidad como especie», una utopía que intenta eliminar ligaduras de género, históricas o sociales y religiosas (Castells, 2002).

\section{Implicaciones}

Se pueden señalar algunas implicaciones que atañen al lenguaje y la ruptura con los vínculos al territorio, a las relaciones entre género, comunidad y tecnología y a la construcción del conocimiento sobre la biodiversidad. En primer lugar, la marcada diferenciación de género entre los casos supone cuestionar cómo afecta a la forma en que se percibe el paisaje. Al analizar el caso de Cerezales vemos que se confirma el papel histórico y cultural protagonista de las mujeres en los ámbitos de la domesticidad, donde se colocan la vegetación, los alimentos, la salud y los cuidados. Además, las oportunidades económicas y jurídicas desiguales hacen crecer el interés en actividades que se oponen a las amenazas de los recursos comunes y esto favorece su papel como relatoras y vinculantes de la comunidad (Shiva, 2003). El orgullo con el que asocian «pueblo» a naturaleza, diversidad alimentaria y conocimientos prácticos refleja su resistencia a la supuesta superioridad urbana. La conversación que se abre provoca la revalorización del medio ambiente cercano, la comunidad reafirma la necesidad de conservar los recursos y los saberes y esto se acompaña de una mejora de la autoestima y del sentido de comunidad. Lejos de identificarse con la madre tierra, sus motivaciones son la responsabilidad personal, la privacidad y los derechos individuales (Rivera Cusicanqui, 2010). Al ir estableciéndose la confianza y la validez del conocimiento popular el grupo intercambia recetas y remedios (Fig. 5) y al final del año se crea una red que trata de mantenerse después del proyecto de investigación.

Si miramos al caso de Valldaura, vemos que se perpetúa la brecha histórica que separa la presencia femenina de la investigación científica y que la cultura técnica masculina es un importante componente "identitario" favorecido por la movilidad geográfica y el nivel de estudios (Chabaud-Rychter, 1997; Berg, 1997; Vergés Bosch, 2012). Barcelona es, además, un contexto privilegiado para el acceso a las tecnologías de la comunicación y Collserola añade posibilidades de intersección entre arte, tecnología y medioambiente. En Opengreens hay una alta presencia de hombres que se mueven de forma itinerante por espacios de intercambio de conocimiento -físicos y virtuales- y que marcan sus propias trayectorias de aprendizaje con el objetivo de crear conocimiento compartido. Sus habilidades con los nuevos medios se materializan fabricando herramientas para uso público, entre ellos artefactos, dispositivos electrónicos, cartografías, prototipos, archivos e interfaces web. El sentido de comunidad que les une se basa en la solidaridad de los movimientos sociales y sus motivaciones, entre utilitaristas y creativas, están marcadas por la curiosidad, las 
expectativas laborales, el placer de experimentar en un entorno amigable, la sociabilidad y las posibilidades de crear contenidos para las redes (Vergés Bosch, 2012).

Figura 5. Artefactos intercambiados por los miembros del Caso 1.

Fuente: Fundación Cerezales, archivo de Econodos.

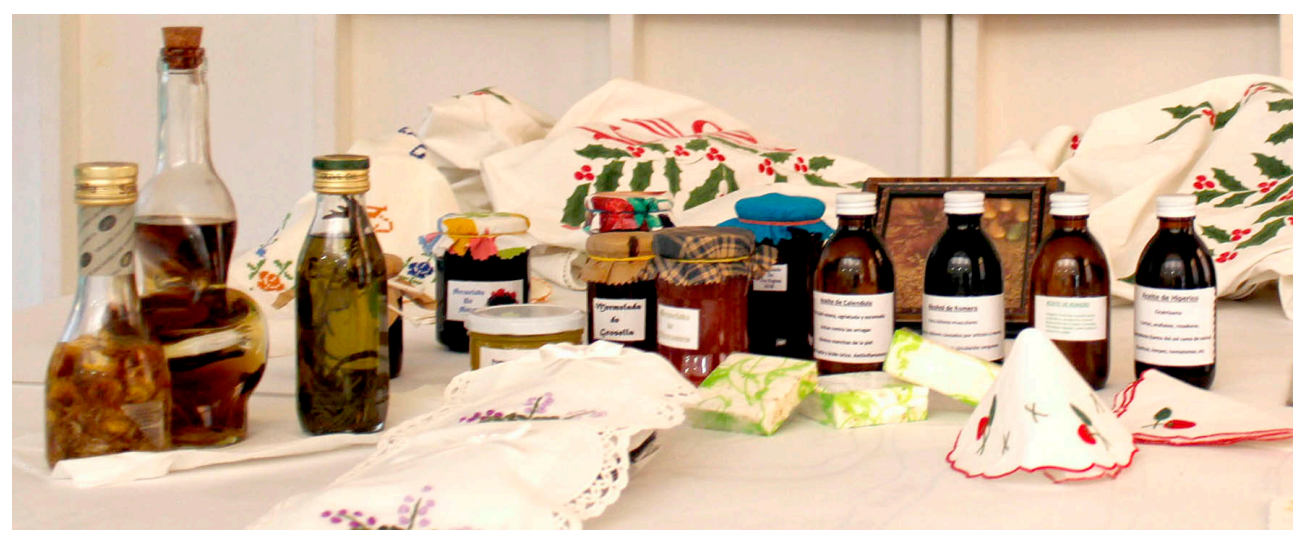

En segundo lugar, el acercamiento a la biodiversidad en Herbarium produjo un sentimiento generalizado de redescubrimiento de las plantas medicinales. Los visitantes y nuevos habitantes alimentan su curiosidad sobre los usos de las plantas acudiendo a medios contemporáneos como libros o Internet, que, aunque implique malentendidos, ofrece datos exhaustivos sobre sustancias medicinales y formas de cultivo y puede llegar a formar parte de la tradición local. Algunas oriundas aprecian la extinción de especies comestibles y medicinales salvajes y relatan la forma en que son usadas para la curación de personas y de animales. Perciben el paisaje desde la necesidad de curar o cuidar a alguien y consideran la flora local una fuente de "materia médica" o "herbal landscape" lo que revela complejas motivaciones e interacciones locales y ecológicas (Soukand y Kalle, 2010). Es un conocimiento transmitido de forma personal y oral, a menudo in situ donde crecen las plantas y su base empírica se fundamenta en la observación del medio y en criterios de acoplamiento a los ciclos naturales, un pensamiento que Nila Gutiérrez, vecina del pueblo, expresa así: -Eso cuando no puedes con ello "déjalo pa prao", que esté unos años dando yerba y después volver a sembrar, porque ya son muchos años sembrando lo mismo y yo creo que la tierra se cansa.

De forma diferente, los vecinos/as de Valldaura señalan con orgullo las especies autóctonas silvestres y cultivadas y la conversación provoca el debate sobre las políticas de su gestión, uso y conservación. Mientras, el colectivo Opengreens hace una crítica al mercado de la alimentación y señala especies culinarias relacionadas con su infancia, el bienestar y el sabor o, incluso, plantas imaginarias. Su gran preocupación es la crisis de las abejas y la polinización a nivel planetario, por lo que uno de sus proyectos más importantes es la construcción de colmenas monitorizadas con las que pretenden contribuir a la búsqueda de soluciones a esa problemática (Figura 6A, 6B). Su conocimiento de los fenómenos naturales proviene de la educación superior, 
lecturas en Internet y aprendizaje compartido y perciben el paisaje como fuente de información e investigación. El colectivo se basa en la total confianza en el método y el empirismo científico, lo cual se hace explícito en la expresión de Jonathan Michin, coordinador de algunas actividades en Valldaura Labs, cuando dice "Agricultura de precisión... un sistema de precisión como experiencia y podemos llegar a un "commons" otra vez".

Figura 6A, 6B. colmenas con dispositivos de videocámara diseñadas y construidas por Opengreens, 2015. Fuente: archivo de Econodos.
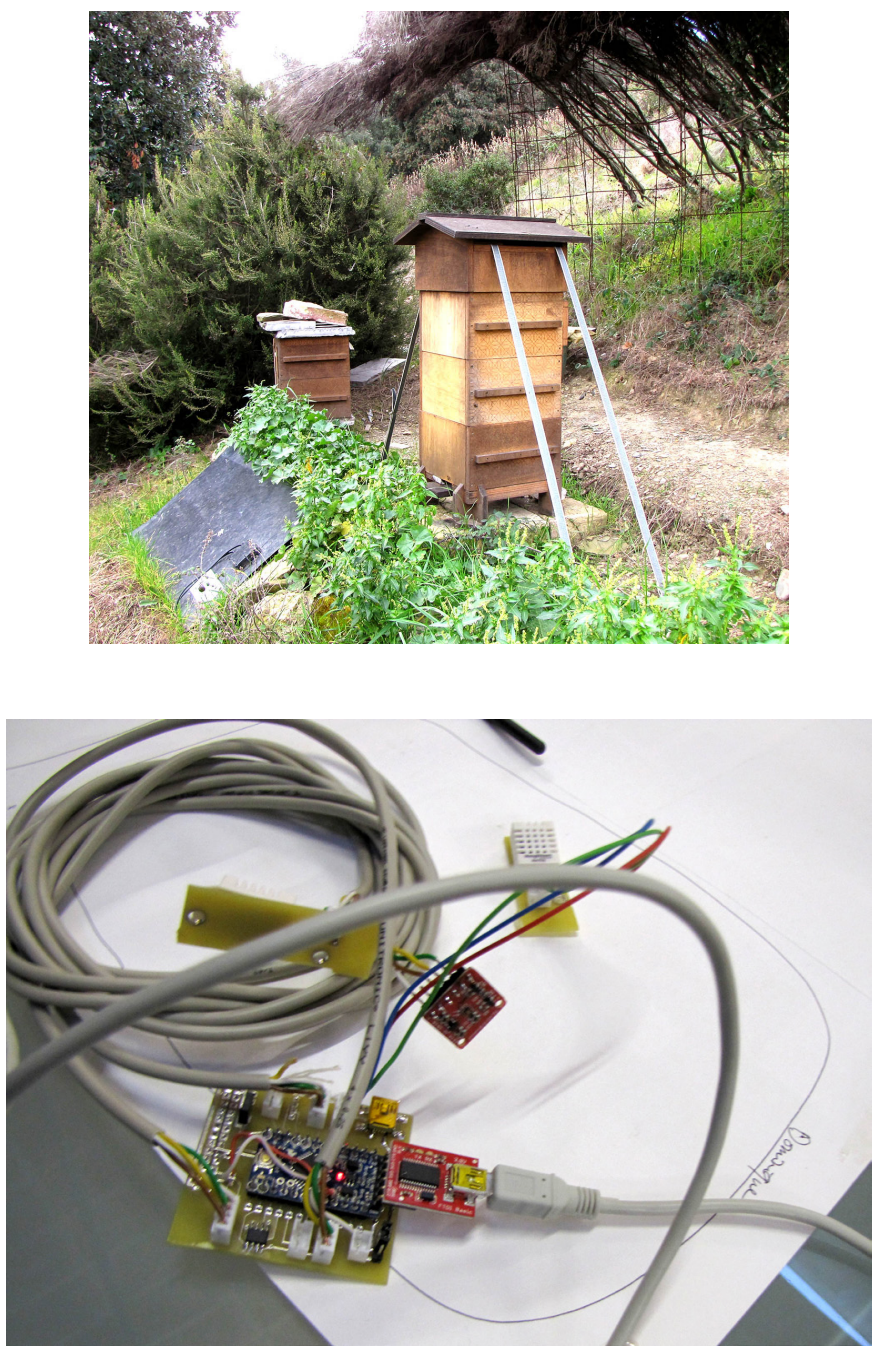

En tercer lugar, el estudio pone en evidencia el profundo ejercicio social y político que supone el uso del lenguaje. Esto se observa en la insistencia generalizada en la identificación de las plantas a través de los nombres latinos en Herbarium y el uso de la lengua inglesa en las producciones de Opengreens. En busca de un espacio 
franco de entendimiento y de legitimidad, ambas lenguas son un instrumento oficial para nombrar y clasificar la biodiversidad de todo el planeta y para facilitar el desarrollo de la investigación científica. Sin embargo, el conocimiento sobre la biodiversidad mediado por el lenguaje científico genera la deslocalización y desterritorialización del conocimiento local, perdiendo infinitos sistemas de nomenclatura local y taxonomías nativas (Schlesinger, 2004). La historia lo refleja durante la Europa medieval, cuando el rico conocimiento empírico de campesinas y herbolarias laicas perseguidas durante la caza de brujas y el de los nativos americanos de las colonias españolas y portuguesas fue expropiado por académicos naturalistas del Imperio español y portugués (Federici, 2004). Algo que no habría sido posible sin el sistema de nomenclatura de Linneo que, a partir del siglo XVII, se convirtió en el instrumento oficial para nombrar y clasificar la vegetación en todo el planeta, facilitando así el desarrollo de algunas metrópolis europeas (Schiebinger y Swan, 2005).

Esto se traduce hoy en día en una tensión global/local que, entre otros aspectos, se puede observar en el creciente interés de las comunidades nativas por las esferas globales, lo que provoca la difusión del conocimiento local en espacios y audiencias diferentes a los de sus orígenes. Estos saberes pueden adoptar un estatus global reconfigurando su valor, sentido simbólico y económico lo que, a su vez, transforma los cultivos en productos y fuentes de riqueza generando conflictos sobre derechos de propiedad intelectual y de patrimonio cultural y natural (Bayardo y Spadafora, 2001). Muchas veces, los discursos dominantes ensombrecen las economías locales y las redefiniciones de género y del medioambiente que puedan estar emergiendo llegando a la depredación de espacios de vida (Escobar, 1999). De hecho, las políticas de la naturaleza y la cultura pueden conducir al desmembramiento de las culturas campesinas mediante categorizaciones fáciles que fabrican un multiculturalismo ornamental y simbólico. Algunos casos de 2etnoturismo" y "ecoturismo" ponen en juego la teatralización del origen como algo anclado en el pasado, lo que dificulta su inclusión como sujeto de la historia (Cusicanqui, 2010).

\section{Conclusiones. Comparando lo incomparable}

La globalización conlleva a deshacerse de fronteras y de límites históricos y, a pesar de la disparidad de los casos presentados, su comparación permite pensar más allá de una diferencia y conservar el detalle del fenómeno sin atender a la escala (Strathern 1994). Así, nos situamos en un escenario en el que el orden tradicional del espacio basado en una clara jerarquía entre lo local, lo nacional y lo internacional está mutando. Las identidades campesinas son minoritarias y las nuevas formas de colonización del medio rural buscan en el paisaje una fuente de bienestar e interés intelectual. La convivencia entre oriundos, visitantes y nuevos habitantes confronta los idearios del éxodo ilustrado, el idilio rural y el retorno ilustrado. Frente a este contexto, el dilema generacional es abordado en Herbarium tratando de facilitar la transmisión de conocimientos y ahondando en el dominio etnográfico de la cultura popular en relación a la ciencia. Sin idealizar el conocimiento local, es una oportunidad de crear redes propias de acceso a recursos u oportunidades, mientras proponen modelos de relaciones sociales y de hibridación cultural que mezclan lo moderno con lo tradicional y lo local con 
lo transnacional. La gestión de la biodiversidad, la alimentación y la salud como formas de «"erritorialización" revelan que el sentido del lugar y su expresión en forma de lealtades socioculturales y afectivas emerge a nivel local. Por otro lado, el intercambio de información, ideas y metodologías y las interfaces digitales supone la construcción de nuevos paradigmas de relación, comunicación y acción social en Opengreens. Su actividad es esencial para la construcción de bienes y recursos que conforman el tejido cultural de redes a gran escala (Marín García, 2013). Sin embargo, dado su fuerte carácter deslocalizado, su integración en las economías y culturas locales es comprometido. La gestión de la información como forma de «territorialización» revela que la identificación con entidades globales como la nación, el planeta o la biosfera requieren de procesos complejos de mediación (Heise, 2013).

Pese a sus diferentes dinámicas, ambos casos comparten una ambigua relación entre biodiversidad y tecnología que evidencia transformaciones del medioambiente pero que mantiene fuertes asimetrías históricas de género. Confluyen en una crítica a la cultura de "usar y tirar" y reafirman la capacidad de que los objetos pueden ser reusados y reparados y las posibilidades del "hazlo tú mismo". Además, el primero subraya el hecho de que las culturas de lo natural han de ser forjadas de acuerdo a procesos empíricos de aprendizaje de los fenómenos biofísicos, mientras que el segundo advierte que lo digital no sustituye lo orgánico. La historia demuestra que la relevancia de una determinada tecnología y su aceptación por la sociedad es producto de la apropiación que los individuos hacen de ella para satisfacer sus necesidades y fortalecer las reivindicaciones locales (Castells 2009; Micarelli 2010; Gyekye 2010). Los artistas que trabajan con los nuevos medios pueden contribuir a crear nuevas tecnologías y posibilidades culturales, pero también son llamados a dar un paso atrás y usar su conocimiento para abordar de forma crítica las implicaciones no representadas y a identificar tendencias futuras más allá de sus confines utilitarios (Wilson, 2002). Su actividad puede enriquecer el carácter testimonial de los conocimientos para hacerlos visibles y crear procesos profundos de territorialización. Las estrategias de comunicación, documentación y archivo, la generación de subjetividades y experiencias estéticas, así como el cultivo de aptitudes tecnológicas apropiadas pueden ser los motores de nuevos sentidos de comunidad y de procesos de construcción colectiva del conocimiento. Es ahí donde la práctica artística cobra su sentido, en la voluntad y oficio de los creadores como traductores interculturales capaces de manejar diferentes lenguajes y categorías, universos simbólicos y aspiraciones. Se trata de un reto al rol de la imaginación que Appadurai (2001, citado en Moraga, 2012) considera fruto de los flujos globales de personas y de contenidos mediáticos y que Santos y Meneses (2010) reclaman en la búsqueda de una "ecología de saberes" capaz de rehacer múltiples experiencias epistemológicas.

\section{Agradecimientos}

A la Fundación Cerezales Antonino y Cinia, a la organización Valldaura Laboratorios de Autosificiencia, a la asociación Econodos, a la Universidad de Oviedo, a la organización Okno y al Parque Natural de Collserola. 


\section{Referencias}

Alberich González, J., (2011). La metropolitanización del territorio catalán: un análisis a partir de los espacios de vida de la población. Población y Espacios Urbanos. Departamento de Geografía Humana, UB y Grupo de población de la AGE, 297-312. Recuperado de: http://www.ub.edu/congreso_poblacion/docs/actas.pdf.

Baigorri, L. (2003). Recapitulando: modelos de artivismo (1994-2003). Artnodes, 1-12. Recuperado de: https://artnodes.uoc.edu/espai/esp/art/baigorri0803/baigorri0803.html

Bayardo R., y Spadafora, A. M. (2001). Derechos culturales y derechos de propiedad intelectual: un campo de negociación conflictivo. Zamudio, T., Dir. Cuadernos de Bioética 7, 1-14. Argentina: Ad Hoc Ed. Recuperado de: http://indigenas.bioetica.org/not/PDF/ Bayardo-Spadafora.pdf

Belá, G. (2012). Ecology as Cultural Critique, Ecology as Cultural Change. Bruselas: OKNO.

Blanchar, C. (2015, 15 de febrero). Collserola perfila su futuro. El País. Recuperado de: http://ccaa.elpais.com/ccaa/2015/02/15/catalunya/1424029211_750724.html.

Benito Lucas, D. (2013). Despoblación, desarraigo y escuela rural: condenados a encontrarse. Encrucijadas. Revista Crítica de Ciencias Sociales 6, 56-69. Recuperado de:

http://www.encrucijadas.org/index.php/ojs/article/view/49.

Boixo, G. (2002). Apuntes para la historia de Vegas del Condado. Web del Ayuntamiento de Vegas. Recuperado de: http://www.vegasdelcondado.com/apuntes.htm\#Presentaci\%F3n].

Cabañes Martínez, E. (2013). De la hibridación al procomún: construyendo la realidad a través de la tecnología. Arsgames, Centro Multimedia del CENART. Jóvenes, tecnofilosofía $y$ arte digital. Revista de Estudios de Juventud, 102, 9-23. Recuperado de: http://euridicecabanes.es.tl/De-la-hibridaci\%F3n-al-procom\%FAn.htm.

Carrero de Roa, M. (2010). Apuntes del curso guías de interpretación del paisaje natural y rural de Asturias. Cecodet, centro de desarrollo territorial de la Universidad de Oviedo.

Castells, M. (2002). El reverdecimiento del yo: el movimiento ecologista. En Castells, M. La Era de la Información: El Poder de la Identidad (2ª ed., 1ª ed. 1998). Vol. II, 135-158. México, Distrito Federal: Siglo XXI.

Castells, M. (2009). Reprogramando las redes de comunicación: movimientos sociales, política insurgente y el nuevo espacio público. En Castells, M. (2009). Comunicación y Poder, 393-533. Madrid: Alianza.

De Vicente, J. (2011). En el espacio exterior. Revista Zehar, 164-175.

Douglas, A. M. (2004). On the Edge: An exploration of the Visual Arts in Remote Rural Contexts on Northern Scotland. Miles, M. \& Hall, T. (Eds), Interventions: Art and Urban Futures 4, 89-106. Portaln USA and Bristol: Intellect.

Duxbury, N. \& Campbell, H. (2009). Developing and Revitalizing Rural Communities Through Arts and Creativity: A Literature Review. Centre for Policy Research on Culture and Communities Simon Fraser University. Prepared for the Creative City Network of Canada.

Eiroa, T. (2013). Paisaje rural: imagen e identidad. Recuperado de: http://elblogdefarina.blogspot.com.es/2013/10/paisaje-rural-imagen-e-identidad.html.

Escobar, A. (1994). Welcome to Cyberia. Notes on the Anthropology of Cyberculture. Current Anthropology, 35, (3), 211-231. Recuperado de: https://www.jstor.org/ stable/2744194.

Escobar, A. (1999). After Nature. Steps on antiessencialist political ecology. Current Antrophology 40 (1), 1-30. DOI: https://doi.org/10.1086/515799 
Esparcia, J. B. (2005). New Rural-Urban Relationships in Europe: A Comparative Analysis. Experiences from The Netherlands, Spain, Hungary, Finland and France. Valencia: Instituto Interuniversitario de Desarrollo Local, Universidad de Valencia.

Greenfield, P. (2009). Linking social change and developmental change: Shifting pathways of human development. Developmental Psychology (45), 401-418. Doi: 10.1037/a0014726

Heise, U. K. (2013). From the Blue Planet to Google Earth. Journal e-flux, 50. Recuperado de: http:/www.e-flux.com/journal/from-the-blue-planet-to-google-earth/.

Federici, S. (2004). Calibán y la bruja. Mujeres, cuerpo y acumulación originaria. Madrid: Traficantes de Sueños.

Fernández-Catuxo, J. (2014). ¿Qué queda de nuestros pueblos? Recuperado de: http://supraterram.wordpress.com/.

García Ramón, M. D. et al. (1995). Mujer y agricultura en España: género, trabajo y contexto regional. Barcelona: Oikos-Tau.

Graña, F. (2004). Ciencia y tecnología desde una perspectiva de género. Documento de trabajo editado por la Facultad de Humanidades y Ciencias de la Educación (UDELAR, Montevideo); informe de avance del proyecto Constitución y reproducción de estereotipos masculinos en el aula.

Gyekye, K. (1995). Technology and Culture in a Developing Country. En Fellow, R. et al. Philosophy of Thecnology 38, 1-5. Cambridge: Cambridge University Press.

Izquierdo, J. (2011). La casa de mi padre. Oviedo: KRK Ed.

Jovchelovitch, S. (2007). Knowledge in context: representations, community and culture, vol. XII. London: Routledge.

Kuznetsov, S. et al. (2010). Rise of the Expert Amateur: DIY Projects, Communities, and Cultures. Proceedings: NordiCHI 2010, 16-20. DOI: 10.1145/1868914.1868950

Lafuente, A. y Valverde, N. (2005). Linnaean Botany and Spanish Imperial Biopolitics, 134147, en Schiebinger, L. et al. (2005). Colonial Botany: Science, Commerce, and Politics in the Early Modern World. Philadelphia: PA, University of Pennsylvania Press.

Leavy, P. (2008). Method Meets Art-arts based research practice. New York: Guildford Press.

Le Men, J. (2002). Léxico del Leonés actual. Colección Fuentes y Estudios de Historia Leonesa. León: Centro de Estudios e Investigación San Isidoro, 743-746.

Lévi-Strauss, C. (2002). El pensamiento salvaje (1 ${ }^{\mathrm{a}}$ ed. 1962). Madrid: Fondo de Cultura Económica S.L.

Luginbühl, Y. (2008). Representaciones sociales del paisaje y sus evoluciones. En Maderuelo D. J., Centro de Arte y Naturaleza de la Fundación Beulas, Paisaje y territorio, 143-180. Madrid: ABADA Editores.

Luginbühl, Y. (2008). Paisaje y bienestar individual y social. Nogué, J. P. (2008). Paisatge $i$ Salut, Vol. 1, 16-35. Olot: Observatori del Paisatge de Catalunya; Barcelona: Generalitat de Catalunya. Departament de Salut.

Lynch, K. (1960). The image of the city. Cambridge MA: MIT Press.

Marín García, T. et al (2013). Los colectivos artísticos: microcosmos y motor del procomún de las artes. Teknokultura: Revista de Cultura digital y Movimientos sociales, 10 (1), 49-74. Recuperado de: http://revistas.ucm.es/index.php/TEKN/article/view/48054/44931

Micarelli, G. (2010). Pensar como un enjambre. En Chaves, M. Perspectivas antropológicas sobre la Amazonía contemporánea. Bogotá: Instituto Colombiano de Antropología e Historia y Pontificia Universidad Javeriana.

Moraga, R. (2012). Hannerz y Appadurai: la transnacionalidad ¿anda suelta? Revista de Ciencias Sociales 28, 1-22. Recuperado de: http://www.uacm.kirj.redalyc.redalyc.org/ articulo.oa? $\mathrm{id}=70824554012>$ 
Moreno Domínguez, A. (2003). La situación laboral de la mujer rural en Castilla y León y Extremadura: un análisis sociológico. Acciones e Investigaciones Sociales, (17), 109-153. Recuperado de: https://dialnet.unirioja.es/servlet/articulo?codigo $=698108$

Nogué, J. (1992). Turismo, percepción del paisaje y planificación del territorio. Estudios turísticos 115, 45-54. Recuperado de: http://hdl.handle.net/10256/4102

Ortega Cantero, N. (2009). Paisaje e identidad. La visión de Castilla como paisaje nacional. Boletin de la A.G.E. 51, 25-49. Recuperado de: http://age.ieg.csic.es/boletin/51/02ORTEGA.pdf

Pérez Soriano, J. (2013). ¿Por qué se van? Mujeres de pueblo y desarraigo en la ruralidad valenciana. Encrucijadas. Revista Crítica de Ciencias Sociales 6, 101-116. Recuperado de: http://www.encrucijadas.org/index.php/ojs/article/view/51

Quiroga, F. G. (2013). Territorios Comunes: Mundo Rural y Procomún. ENCRUCIJADAS. Revista Crítica de Ciencias Sociales (6), 18-25. Recuperado de: http://www.encrucijadas. org/index.php/ojs/issue/view/3

Rebotier, J. (2012). El investigador, el trabajo de campo y los datos a prueba de la comparación. Ciencias sociales y problemas emergentes: ¿Cómo identificarlos? Bubok,113-126. Recuperado de: https://halshs.archives-ouvertes.fr/halshs-00686456.

Rivera Cusicanqui, S. (2010). Chhixinakax utxiwa. Una reflexión sobre prácticas y discursos descolonizadores. Buenos Aires: Retazos-Tinta Limón. Recuperado de: https://chixinakax. files.wordpress.com/2010/07/silvia-rivera-cusicanqui.pdf

Rofes, O. (2003). Learning from the tourist. The construction of local specifities from artistic activities. Territories en process, 302-302. Madrid: Idensitat e Instituto de la Juventud.

Santos, B. de Sousa, y Meneses, M. P. (2010). Epistemologias do Sul (2 ${ }^{\mathrm{a}}$ ed.). Coimbra: Edições Almedina, CES, Série Conhecimento e Instituições; 2.

Schiebinger, L. (2004). Linguistic Imperialism. En Schiebinger, L. Plants and Empire: Colonial Bioprospecting in the Atlantic World, 194-225. Cambridge: Harvard University Press.

Schiebinger, L., y Swan, C. (2005). Colonial Botany: Science, Commerce, and Politics in the Early Modern World, 134-147. Philadelphia: PA, University of Pennsylvania Press.

Shiva, V. (2003). Cosecha robada: el secuestro del suministro mundial de alimentos. Barcelona: Paidós.

Soukand, R., and Kalle, R. (2010). Herbal landscape: The perception of landscape as a source of medicinal plants. Trames, 14 (64/59), (3), 207-226. Recuperado de:

DOI: http://www.kirj.ee/17748/?tpl=1061\&c_tpl=1064

Strathern, M. (1998-1980). No Nature no Culture: The Hagen case en Nature. En C. U. Maccormack, Ed. Culture, nature and gender, 174-222. Cambridge University Press.

Thapalyal, R. (2005). Notas del curso Shades. Yoruba and Ancient Indian ideas on Creativity, Space and Self, Glasgow School of Art, Historical and Critical Studies. Glasgow, Scotland UK.

Vergés Bosch, N. (2012). Gènere i TIC: el procés d'autoinclusió de les dones en les TIC. Una aproximació des de les tecnòlogues artístiques i les tecnòlogues informàtiques. Tesis de Doctorado. Barcelona: Programa de Doctorat en Societat de la Informació i el Coneixement. Universitat Oberta de Catalunya (UOC). Internet Interdisciplinary Institute (IN3). Programa de Recerca Gènere i TIC.

Welpa, M., de la Vega-Leinerta, A., Stoll-Kleemannb, S. \& Jaegera, C. (2005). Science-based stakeholder dialogues: Theories and Tools. Potsdam Institute for Climate Impact Research (PIK), Department of Global Change \& Social Systems \& Humboldt University of Berlin, Institute of Agricultural Economics and Social Sciences, Berlin. Recuperado de:

DOI: http://dx.doi.org/10.1016\%2Fj.gloenvcha.2005.12.002. 
Wilson, S. (2002). Information arts: intersections of art, science, and technology. Cambridge (Massachusetts) London: MIT Press, Colección: Leonardo.

Yin, R. K. (2003-09). Case Study Research: design and methods. California: SAGE. 